\title{
Posttraumatic Hydrocephalus: Risk Factors, Treatment Modalities, and Prognosis
}

\author{
Vamsi Krishna Yerramneni ${ }^{1}$ Vamsi Krishna Kotha ${ }^{1}$ \\ 1 Department of Neurosurgery, Nizam's Institute of Medical \\ Sciences, Punjagutta, Hyderabad, Telangana, India \\ Indian J Neurosurg 2017;6:198-202.
}

\begin{abstract}
Address for correspondence Vamsi Krishna Yerramneni, MBBS, MCh, Department of Neurosurgery, Nizam's Institute of Medical Sciences, Punjagutta, Hyderabad, Telangana India, 500083 (e-mail: vamsiky.ns@gmail.com).
\end{abstract}

\begin{abstract}
Background Posttraumatic hydrocephalus (PTH) is a rare clinicopathological entity seen as a sequel of head injury ( $\mathrm{HI})$. The described incidence in literature is 7 to $29 \%$. Aims and Objectives To study the incidence, risk factors, treatment options, and prognosis.

Materials and Methods We retrospectively reviewed our $\mathrm{HI}$ database from our institution and identified patients with PTH. The data were obtained from the case files, radiologic records, telephonic interviews, and letters.

Results PTH was diagnosed in 32 patients with mean age being $33.7 \pm 16.0$ years. Severe $\mathrm{HI}$ was present in 22(68.75\%) patients, and all of them underwent decompressive craniectomy (DC). Remaining patients had either cranial base repair for cerebrospinal fluid (CSF) leak or conservative management. Average duration for development of PTH after the $\mathrm{HI}$ was $11.05 \pm 0.58$ months in DC group and $17.0 \pm 1.25$ months in the non-DC group. Patients were treated by CSF diversion (ventriculoperitoneal [VP] or thecoperitoneal shunt) and lumbar subarachnoid drainage in the perioperative period of cranioplasty. Average interval between DC and cranioplasty was $6.21 \pm 14.0$ months.

Keywords

- posttraumatic hydrocephalus

- head injury

- decompressive craniectomy

- cranioplasty

- shunt Mean follow-up was $72.23 \pm 0.67$ months and observed mortality was $32 \%$. Statistical analysis showed correlation between severity of HI, DC, and development of PTH. DC also had correlation with early development of PTH.

Conclusion The incidence of PTH is low and the etiology is multifactorial. Severe HI, DC, and delayed cranioplasty ( $>6$ months) are associated with higher chances of PTH development. Though VP shunt placement is the established treatment modality, temporary lumbar subarachnoid drain placement in perioperative period of cranioplasty in selected cases is an option.
\end{abstract}

\section{Introduction}

Ventriculomegaly following severe head injury (HI) is a well-noted entity, but the diagnostic criteria for labeling it as posttraumatic hydrocephalus (PTH) and the treatment protocol vary among different centers. Consequently, the reported incidence of PTH varies widely from 0.7 to $29 \%$. If computed tomographic (CT) criteria of abnormal ventriculomegaly alone are used, the incidence of PTH is

received

July 18,2017

accepted

August 18, 2017

published online

October 13, 2017
DOI https://doi.org/

10.1055/s-0037-1606613. ISSN 2277-954X. of the order of 30 to $86 \% .^{1}$ The generally accepted method of diagnosing PTH depends on a combination of clinical, imaging, and physiologic data. ${ }^{2,3}$ The clinical diagnosis of PTH is often confounded by the signs and symptoms of secondary brain injuries like hypoxic damage, edema, and infections. Moreover, the poor prognosis associated with the severe HI complicates the management. The authors did a retrospective study to look at various factors associated with PTH and the treatment modalities and outcomes.
(C) 2017 Neurological Surgeons' Society of India
License terms

(๑) $\Theta \circledast$ 


\section{Materials and Methods}

\section{Patient Population}

The data were collected from the institutional HI database from 2004 through 2016. Thirty-two cases with the diagnosis of PTH were identified. Patients' demographic profile, Glasgow coma scale (GCS) at admission, CT findings of the brain, initial surgery performed, clinical features at the time of PTH, and various treatment modalities followed were noted from the admission records. Outcome assessments were done using Glasgow coma outcome score-extended (GOS-E). Follow-up data were obtained from outpatient reviews, telephonic interviews, and death summaries.

\section{Posttraumatic Hydrocephalus Diagnosis Criteria and Radiologic Assessment}

Posttraumatic ventriculomegaly was defined by radiologic evidence of ventricular dilatation, as evidenced by an Evans index (or ratio) of $>0.3$ at 6 months follow-up, and PTH was defined by radiologic evidence of progressive ventricular dilatation with trans-ependymal edema in the form of periventricular lucencies (PVLs). Other radiologic features observed included enlargement of temporal horn, third and fourth ventricles, and the presence of either clinical deterioration or failure to make neurologic progress over time in the absence of other identifiable causes ( - Fig. 1).

\section{Treatment of Posttraumatic Hydrocephalus}

In patients who had flap bulge with ventriculomegaly but no significant periventricular ooze and minimal clinical symptoms, lumbar drain placement was done for 2 days before cranioplasty and retained till second postoperative day. Subsequently they were observed for 4 to 5 days before discharge.
In cases with diagnosed PTH and overt clinical symptoms, ventriculoperitoneal (VP) or thecoperitoneal (TP) shunt placement was done as per the surgeon's preference.

\section{Statistical Analysis}

Statistical analysis was done on SPSS statistics for Windows, software version 20.0 (IBM, Corp.), using independent $t$-test.

\section{Results}

PTH was diagnosed in 32 out of the 1,560 patients with HI treated over the period. The incidence of the PTH is $2.05 \%$, with 27 (84.37\%) males and 5 (15.62\%) females. Mean age was $33.7 \pm 16$ (range: $18-60$ years).

Head injury resulted from road traffic accident in 30 (98.75\%) patients and fall from height in $2(1.25 \%)$ patients. Severe HI (GCS $\leq 8)$ was present in 22 (68.75\%) cases at the time of initial presentation. The CT scan findings showed predominant contusions in 24 (75\%) and contusion and subdural hematoma (SDH) or SDH alone in 10 (31.25\%). Unilateral decompressive craniectomy (DC) was done in 22 (68.75\%) patients, and 4 (12.5\%) patients had anterior cranial fossa defect repair to stop cerebrospinal fluid (CSF) leak.

Out of 22 patients who underwent DC, PTH was diagnosed in $16(72.7 \%)$ patients before cranioplasty. The mean interval between emergency DC and elective cranioplasty was $6.21 \pm 14$ months. The average duration between the $\mathrm{HI}$ and diagnosis of PTH was $11.05 \pm 0.58$ months in patients who had DC and $17.0 \pm 1.25$ months in patients managed conservatively (-Table 1 ).

Independent $t$-test was used to look at correlation between different factors, and a $p$-value $<0.05$ was taken as significant. Age difference was not significant between DC and non-DC group at admission. Severe head injury (GCS $\leq 8$ ), DC, and time duration between $\mathrm{HI}$ and PTH diagnosis
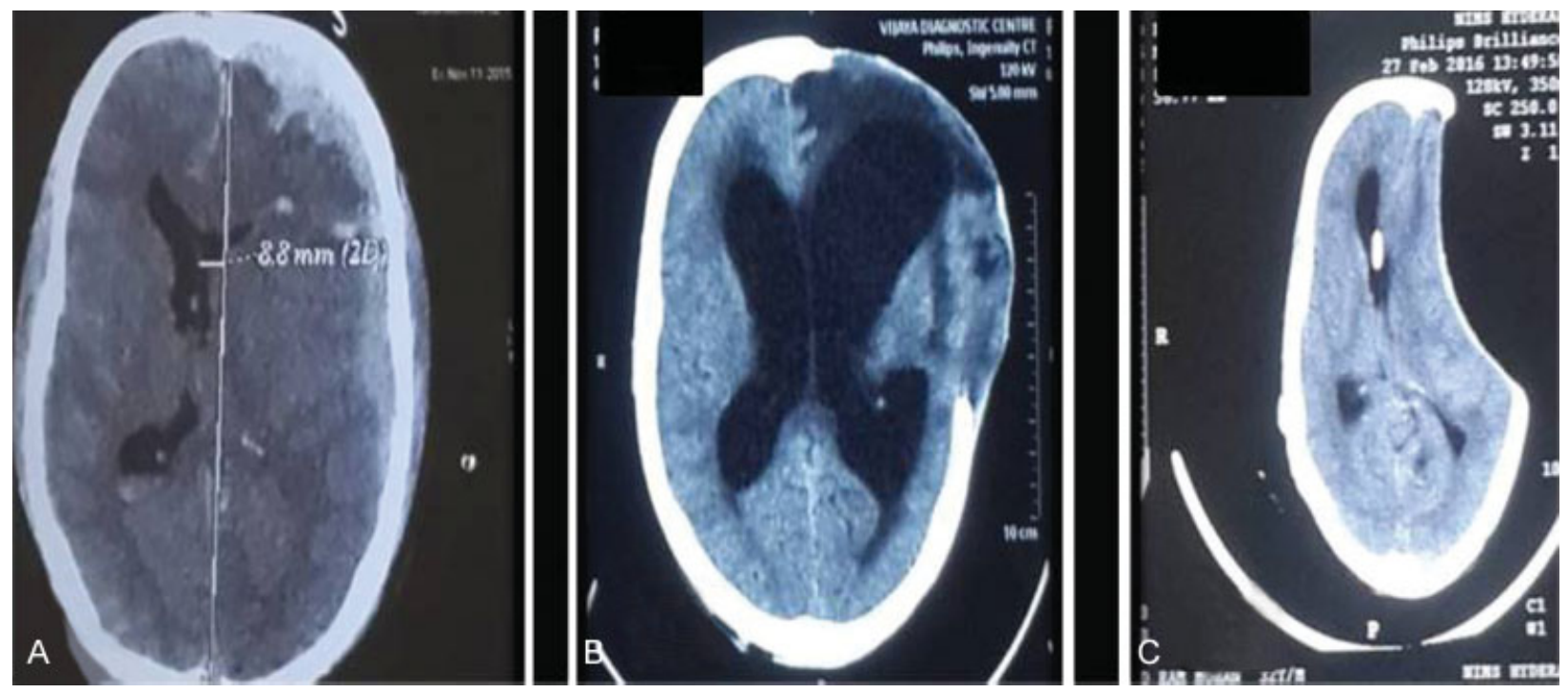

Fig. 1 The CT images of the brain of 32-year-old male patients who had road traffic accident. (A) Plain CT scan of the brain showing left acute subdural hematoma with contusions and midline shift in a 32-year-old man. He underwent left frontotemporoparietal decompressive craniectomy. (B) Postoperative plain CT of the brain after around 4 months showing ventriculomegaly shunt with periventricular ooze and the brain bulge beyond craniectomy defect. The patient underwent VP shunt placement. (C) CT scan of the brain done after 1 week of VP shunt placement shows VP shunt catheter in the lateral ventricle with sunken flap. He subsequently underwent cranioplasty. CT, computed tomography; VP, ventriculoperitoneal. 
Table 1 Various characteristics of the patients

\begin{tabular}{|c|c|c|}
\hline Feature & No. & $\%$ \\
\hline Male & 27 & 84.37 \\
\hline Female & 5 & 15.62 \\
\hline Age (mean \pm SD) & $33.7+16$ & \\
\hline $\begin{array}{l}\text { Severe head injury } \\
(G C S \leq 8)\end{array}$ & 22 & 68.75 \\
\hline \multicolumn{3}{|c|}{ Radiologic features (CT findings of the head) } \\
\hline Contusions & 24 & 75 \\
\hline $\begin{array}{l}\text { Subdural hematoma alone or } \\
\text { with contusions }\end{array}$ & 10 & 31.25 \\
\hline Extradural hematoma & 5 & 15.62 \\
\hline $\begin{array}{l}\text { Depressed fracture and } \\
\text { skull base fractures }\end{array}$ & 7 & 21.87 \\
\hline \multicolumn{3}{|l|}{ Treatment of head injury } \\
\hline DC & 22 & 68.75 \\
\hline $\begin{array}{l}\text { Anterior cranial fossa CSF } \\
\text { leak repair }\end{array}$ & 4 & 12.5 \\
\hline Depressed fracture elevation & 3 & 9.37 \\
\hline Conservative management & 3 & 9.37 \\
\hline \multicolumn{3}{|c|}{$\begin{array}{l}\text { Mean duration between head injury and development of } \\
\text { symptomatic PTH }\end{array}$} \\
\hline In DC group & $11.05 \pm 0.58 \mathrm{mo}$ & \\
\hline In conservative group & $17.0 \pm 1.25 \mathrm{mo}$ & \\
\hline \multicolumn{3}{|c|}{ Clinical features at PTH presentation } \\
\hline $\begin{array}{l}\text { Memory disturbances, gait, } \\
\text { and urinary disturbances }\end{array}$ & 16 & 50 \\
\hline Altered sensorium & 7 & 21.87 \\
\hline $\begin{array}{l}\text { Paraparesis, hemiparesis, } \\
\text { and difficulty walking }\end{array}$ & 4 & 12.5 \\
\hline Loss of consciousness & 1 & 3.12 \\
\hline $\begin{array}{l}\text { Memory disturbances and } \\
\text { slowing of recovery }\end{array}$ & 1 & 3.12 \\
\hline \multicolumn{3}{|l|}{ Treatment for PTH } \\
\hline VP shunt placement & 18 & 56.25 \\
\hline TP shunt placement & 10 & 31.25 \\
\hline $\begin{array}{l}\text { Temporary LP drain } \\
\text { placement in the } \\
\text { preoperative period }\end{array}$ & 4 & 12.5 \\
\hline Mean follow-up period & $72.23 \pm 0.67 \mathrm{mo}$ & \\
\hline No. of patients followed up & 25 & \\
\hline \multicolumn{3}{|c|}{ Average Glasgow coma outcome scale (No. of follow-ups: 25) } \\
\hline Upper good recovery & 6 & \\
\hline Lower good recovery & 4 & \\
\hline Moderate disability & 5 & \\
\hline Lower disability & 2 & \\
\hline Expired during the treatment & 2 & \\
\hline Died during follow-up & 6 & \\
\hline
\end{tabular}

Abbreviations: CSF, cerebrospinal leak; CT, computed tomography; DC, decompressive craniectomy; GCS, Glasgow coma scale; LP, lumbar puncture; PTH, posttraumatic hydrocephalus; SD, standard deviation; TP, thecoperitoneal; VP, ventriculoperitoneal.
Table 2 Difference between DC and non-DC group

\begin{tabular}{|l|l|l|}
\hline & DC & Non-DC \\
\hline Mean age & $29.09 \pm 9.90$ & $25.40 \pm 7.38$ \\
\hline GCS $\leq 8$ & 22 & 0 \\
\hline GCS $>8$ & 0 & 10 \\
\hline $\begin{array}{l}\text { Time duration between } \\
\text { HI and PTH diagnosis } \\
(\text { mo })\end{array}$ & $11.05 \pm 0.58$ & $17.0 \pm 1.25$ \\
\hline
\end{tabular}

Abbreviations: DC, decompressive craniectomy; GCS, Glasgow coma scale; HI, head injury; PTH, posttraumatic hydrocephalus.

have a significant correlation $(p=0.041)$. Patients who underwent DC presented with PTH early compared with conservatively managed patients. The correlation between timing of cranioplasty and development of PTH could not be established because of small sample size ( $\mathbf{-}$ Table 2 ).

The predominant clinical presentation is the triad of memory impairment, gait disturbances, and urinary incontinence observed in 16 (50\%) patients, followed by altered sensorium in 7 (21.87\%) and paraparesis or hemiparesis with difficulty walking in 4 (12.5\%) patients.

\section{Management}

Medium pressure VP shunt placement was done in 21 (53.25\%) patients, 7 (25\%) underwent TP shunt, and 4 (12.5\%) had lumbar drain placement during the perioperative period of cranioplasty. Lumbar drain placement was opted when patients had flap bulge and subtle clinical symptoms with CT demonstrating ventriculomegaly with minimal periventricular ooze.

Among all the cases, three patients had VP shunt infections at the abdominal end; three had TP shunt complications in the form of blockade requiring revisions. Nineteen patients improved in GCS post-shunt.

The average follow-up was 6 years range ( 2 months to 14 years).

The mortality was $25 \%$ with the outcomes measured by Glasgow outcome scales as shown in the -Table 1. Two patients died in the hospital with septicemia and four patients died after discharge from the hospital in poor functional status. The records for exact cause of these deaths could not be obtained.

\section{Discussion}

The clinical entity of PTH was first recognized in 1914 by Dandy and Blackfan who described a case of hydrocephalus developed in the child after fall. ${ }^{3}$ The incidence of PTH in this series is $2.05 \%$, indicating that it is a rare and variable incidence in world literature arising from variable diagnostic criteria. ${ }^{2-7}$ The authors used a combination of the clinical and radiologic observations to diagnose PTH. This is more appropriate keeping in view of management strategy and also considering the fact that DC results in ventriculomegaly over a period of time, which may not always be symptomatic. ${ }^{2,3,5,6,8}$ 
PTH diagnosis in this series ranged from 3 weeks to 33 months following head injury and is early in post-DC. Kishore et al reported that within 2 weeks of traumatic brain injury (TBI) $>93 \%$ of cases presented with ventriculomegaly. In another study, Marmarou et al suggested that most cases of ventriculomegaly will present within 1-month postinjury. ${ }^{9,10}$

Some studies have described convexity subdural CSF collection in patients after the DC, before development of hydrocephalus. ${ }^{11,12}$

Predominant presentation -in this series was the triad of symptoms ( $\mathbf{- T a b l e ~} \mathbf{1}$ ) resembling normal-pressure hydrocephalus (NPH) whereas the clinical features of presentation in most of the studies included continuous headaches, morning vomiting, dementia, incontinence, difficulty in walking, papilledema, altered sensorium, failure to improve, and psychomotor retardation. ${ }^{13,14}$

DC seems to have a strong correlation with the development of PTH as observed in this study and is described in the literature. Various factors have been described in the literature including debris causing arachnoid granulations block and change in CSF flow disturbances. $8,9,14-16$

Another finding of this study is that DC also hastens the development of PTH. Though the study could not establish a relationship between timing of cranioplasty and development of hydrocephalus, we strongly believe that delay in cranioplasty may increase the chances of the development of PTH by aggravating the disturbances in the CSF flow dynamics. ${ }^{17}$ However, there is paucity of literature regarding the timing of cranioplasty contributing to the development of PTH. Studies by Chang et al, Chun and Yi, and Schuss et al reported that the early cranioplasty was associated with higher rates of infection. However, delay in cranioplasty might delay progression of neurologic outcome due to numerous factors. The study did not make specific references to PTH as the cause of poor neurologic outcome. ${ }^{18-20}$

Another distinct feature of our study is the treatment of the PTH with lumbar drain placement in the perioperative period of cranioplasty in selected patients. These patients have large subdural collections, resulting in bulging flap with ventriculomegaly and mild periventricular ooze, with only subtle symptoms. This clinical-radiologic picture probably represents the early stages of $\mathrm{PTH}$ given the fact that the subdural CSF collections have been described in the literature as a prelude to the development of PTH. ${ }^{11,12}$ This transient CSF diversion obviated the need for permanent ventricular shunts and needs further study to firmly establish it as a modality of treatment. We used medium-pressure shunts to reduce the risk of extradural hematoma (EDH), SDH, and slit ventricle. ${ }^{19}$ Programmable valve shunt devices are always an option, but high cost prohibits their frequent usage. The incidence of shunt obstruction was associated more with the TP shunts, where shunt functioning is not easily verifiable. Accordingly, there is tendency to use VP shunts toward the later part of the study, and we recommend preference to VP shunt usage. Endoscopic third ventriculostomy (ETV) was performed for PTH with successful outcomes in some studies. ${ }^{1,21,22}$ De Bonis et al, in their review of few published cases of use of ETV for
PTH, concluded that the procedure has a high success rate, especially for patients with elevated intracranial pressure ${ }^{23}$ The mortality is high in patients developing PTH as is evident from this study ( $n=32$, of which eight expired [25\%]). It may be because of the very fact that most of the patients developed PTH as a result of severe head injury and were moribund at the time of diagnosis of PTH. Patients in better preoperative clinical condition tend to have better outcomes. ${ }^{8,24}$

\section{Conclusion}

The incidence of PTH is low and the etiology is multifactorial. Severe HI, DC, and delayed cranioplasty (> 6 months) are associated with higher chances of PTH development. Though VP shunt placement is the established treatment modality, temporary lumbar subarachnoid drain placement in perioperative period of cranioplasty in selected cases is an option requiring further study.

\section{Conflict of Interest}

None.

\section{References}

1 Lee SH, Kong DS, Seol HJ, Shin HJ. Endoscopic third ventriculostomy in patients with shunt malfunction. J Korean Neurosurg Soc 2011;49(04):217-221

2 Guyot LL, Michael DB. Post-traumatic hydrocephalus. Neurol Res 2000;22(01):25-28

3 Licata C, Cristofori L, Gambin R, Vivenza C, Turazzi S. Posttraumatic hydrocephalus. J Neurosurg Sci 2001;45(03):141-149

4 Dandy W, Blackman K. Internal hydrocephalus: an experimental, clinical and pathological study. Am J Dis Child 1914;8:406-482

5 Choi I, Park HK, Chang JC, Cho SJ, Choi SK, Byun BJ. Clinical factors for the development of posttraumatic hydrocephalus after decompressive craniectomy. J Korean Neurosurg Soc 2008; 43(05):227-231

6 Guyot LL, Michael DB. Post-traumatic hydrocephalus. Neurol Res 2000;22(01):25-28

7 Mazzini L, Campini R, Angelino E, Rognone F, Pastore I, Oliveri G. Posttraumatic hydrocephalus: a clinical, neuroradiologic, and neuropsychologic assessment of long-term outcome. Arch Phys Med Rehabil 2003;84(11):1637-1641

8 Sarkari A, Gupta DK, Sinha S, Kale SS, Mahapatra AK. Post-traumatic hydrocephalus: presentation, management and outcome-an apex trauma centre experience. Indian J Neurotrauma 2010;7(02): 135-138

9 Kishore PR, Lipper MH, Miller JD, Girevendulis AK, Becker DP, Vines FS. Post-traumatic hydrocephalus in patients with severe head injury. Neuroradiology 1978;16:261-265

10 Marmarou A, Foda MA, Bandoh K, et al. Posttraumatic ventriculomegaly: hydrocephalus or atrophy? A new approach for diagnosis using CSF dynamics. J Neurosurg 1996;85(06):1026-1035

11 Honeybul S, Ho KM. Incidence and risk factors for post-traumatic hydrocephalus following decompressive craniectomy for intractable intracranial hypertension and evacuation of mass lesions. J Neurotrauma 2012;29(10):1872-1878

12 Kawaguchi T, Fujita S, Hosoda K, Shibata Y, Komatsu H, Tamaki N. Treatment of subdural effusion with hydrocephalus after ruptured intracranial aneurysm clipping. Neurosurgery 1998; 43(05):1033-1039

13 Tian HL, Xu T, Hu J, Cui YH, Chen H, Zhou LF. Risk factors related to hydrocephalus after traumatic subarachnoid hemorrhage. Surg Neurol 2008;69(03):241-246, discussion 246 
14 Cardoso ER, Galbraith S. Posttraumatic hydrocephalus-a retrospective review. Surg Neurol 1985;23(03):261-264

15 Waziri A, Fusco D, Mayer SA, McKhann GM II, Connolly ES Jr. Postoperative hydrocephalus in patients undergoing decompressive hemicraniectomy for ischemic or hemorrhagic stroke. Neurosurgery 2007;61(03):489-493, discussion 493-494

16 De Bonis P, Pompucci A, Mangiola A, Rigante L, Anile C. Posttraumatic hydrocephalus after decompressive craniectomy: an underestimated risk factor. J Neurotrauma 2010;27(11): 1965-1970

17 Beauchamp KM, Kashuk J, Moore EE, et al. Cranioplasty after postinjury decompressive craniectomy: is timing of the essence? J Trauma 2010;69(02):270-274

18 Chang V, Hartzfeld P, Langlois M, Mahmood A, Seyfried D. Outcomes of cranial repair after craniectomy. J Neurosurg 2010; 112(05):1120-1124

19 Chun HJ, Yi HJ. Efficacy and safety of early cranioplasty, at least within 1 month. J Craniofac Surg 2011;22(01):203-207
20 Schuss P, Vatter H, Marquardt G, et al. Cranioplasty after decompressive craniectomy: the effect of timing on postoperative complications. J Neurotrauma 2012;29(06):1090-1095

21 Rangel-Castilla L, Barber S, Zhang YJ. The role of endoscopic third ventriculostomy in the treatment of communicating hydrocephalus. World Neurosurg 2012;77(3-4):555-560

22 Naftel RP, Reed GT, Kulkarni AV, Wellons JC III. Evaluating the Children's Hospital of Alabama endoscopic third ventriculostomy experience using the Endoscopic Third Ventriculostomy Success Score: an external validation study. J Neurosurg Pediatr 2011; 8(05):494-501

23 De Bonis P, Tamburrini G, Mangiola A, et al. Post-traumatic hydrocephalus is a contraindication for endoscopic thirdventriculostomy: isn't it? Clin Neurol Neurosurg 2013;115(01): 9-12

24 Tribl G, Oder W. Outcome after shunt implantation in severe head injury with post-traumatic hydrocephalus. Brain Inj 2000; 14(04):345-354 